\title{
ON WELL-POSEDNESS OF THE NONLOCAL BOUNDARY VALUE PROBLEM FOR PARABOLIC DIFFERENCE EQUATIONS
}

\author{
A. ASHYRALYEV, I. KARATAY, AND P. E. SOBOLEVSKII
}

Received 21 March 2004

We consider the nonlocal boundary value problem for difference equations $\left(u_{k}-u_{k-1}\right) / \tau$ $+A u_{k}=\varphi_{k}, 1 \leq k \leq N, N \tau=1$, and $u_{0}=u_{[\lambda / \tau]}+\varphi, 0<\lambda \leq 1$, in an arbitrary Banach space $E$ with the strongly positive operator $A$. The well-posedness of this nonlocal boundary value problem for difference equations in various Banach spaces is studied. In applications, the stability and coercive stability estimates in Hölder norms for the solutions of the difference scheme of the mixed-type boundary value problems for the parabolic equations are obtained. Some results of numerical experiments are given.

\section{Introduction}

In [6], the coercive stability estimates in Hölder norms for the solution of the nonlocal boundary value problem

$$
v^{\prime}(t)+A v(t)=f(t), \quad 0 \leq t \leq 1, \quad v(0)=v(\lambda)+\varphi, \quad 0<\lambda \leq 1,
$$

in arbitrary Banach space $E$ with the strongly positive operator $A$ were proved. The exact Shauder's estimates in Hölder norms of solution of the boundary value problem on the range $\left\{0 \leq t \leq 1, x \in \mathbb{R}^{n}\right\}$ for $2 m$-order multidimensional parabolic equations were obtained. In [5], the convergence estimates for the solution of first-order accuracy implicit Rothe difference scheme

$$
\begin{gathered}
\frac{u_{k}-u_{k-1}}{\tau}+A u_{k}=\varphi_{k}, \quad \varphi_{k}=f\left(t_{k}\right), 1 \leq k \leq N, N \tau=1, \\
u_{0}=u_{[\lambda / \tau]}+\varphi
\end{gathered}
$$

for approximate solution of this boundary value problem were obtained. We are interested in studying the well-posedness of this difference nonlocal boundary value problem (1.2) in various Banach spaces.

Applying the method of $[9,11,14]$, the well-posedness of difference problem (1.2) in various Banach spaces is studied. In applications, the stability and coercive stability estimates in Hölder norms for the solutions of the difference schemes of the mixed-type 
274 On well-posedness of the nonlocal boundary value problem

boundary value problems for the parabolic equations are obtained. Some results of numerical experiments are given. Finally, notice that the well-posedness of differential and difference equations of the parabolic type has been developed extensively, see, for instance, $[4,10,12]$.

\section{The estimate of stability}

Assume that $A$ is the strongly positive operator, that is, $-A$ is the generator of the analytic semigroup $\exp \{-t A\} \quad(t \geq 0)$ of the linear bounded operators with exponentially decreasing norm.

Let $F_{\tau}(E)$ be the linear space of mesh functions $\varphi^{\tau}=\left\{\varphi_{k}\right\}_{1}^{N}$ with values in the Banach space $E$. Next, on $F_{\tau}(E)$ we denote $C_{\tau}(E)$ - and $C_{\tau}^{\alpha}(E)$-Banach spaces with the norms

$$
\begin{aligned}
\left\|\varphi^{\tau}\right\|_{C_{\tau}(E)} & =\max _{1 \leq k \leq N}\left\|\varphi_{k}\right\|_{E}, \\
\left\|\varphi^{\tau}\right\|_{C_{\tau}^{\alpha}(E)} & =\left\|\varphi^{\tau}\right\|_{C_{\tau}(E)}+\max _{1 \leq k<k+r \leq N}\left\|\varphi_{k+r}-\varphi_{k}\right\|_{E} \frac{(k \tau)^{\alpha}}{(r \tau)^{\alpha}} .
\end{aligned}
$$

The nonlocal boundary value problem $(1.2)$ is said to be stable in $F_{\tau}(E)$ if we have the inequality

$$
\left\|u^{\tau}\right\|_{F_{\tau}(E)} \leq M\left[\|\varphi\|_{E}+\left\|\varphi^{\tau}\right\|_{F_{\tau}(E)}\right]
$$

where $M$ is independent not only of $\varphi, \varphi^{\tau}$ but also of $\tau$.

TheOREm 2.1. Let $\tau$ be a sufficiently small number. Then the boundary value problem (1.2) is stable in $C_{\tau}(E)$ and $C_{\tau}^{\alpha}(E)$.

Proof. The stability of the boundary value problem (1.2) in $C_{\tau}(E)$ was obtained in [5]. The proof of this result is based on the stability in $C_{\tau}(E)$ of the initial value problem for difference equations

$$
\frac{u_{k}-u_{k-1}}{\tau}+A u_{k}=\varphi_{k}, \quad 1 \leq k \leq N, N \tau=1,
$$

$u_{0}$ is given and also the estimate

$$
\left\|u_{0}\right\|_{E} \leq M\left[\|\varphi\|_{E}+\left\|\varphi^{\tau}\right\|_{C_{\tau}(E)}\right]
$$

for solutions of the boundary value problem (2.3), when $\tau$ is a sufficiently small number. If $\tau$ is a sufficiently small number, then from the last estimate for solutions of the boundary value problem (2.3) it follows that

$$
\left\|u_{0}\right\|_{E} \leq M\left[\|\varphi\|_{E}+\left\|\varphi^{\tau}\right\|_{C_{\tau}^{\alpha}(E)}\right]
$$


Finally, from the last estimate for solutions of the boundary value problem (2.3) and the stability in $C_{\tau}^{\alpha}(E)$ of the initial value problem (2.3), the stability in $C_{\tau}^{\alpha}(E)$ of the boundary value problem (1.2) follows. Theorem 2.1 is proved.

The nonlocal boundary value problem (1.2) is said to be coercively stable (well posed) in $F_{\tau}(E)$ if we have the coercive inequality

$$
\left\|\left\{\tau^{-1}\left(u_{k}-u_{k-1}\right)\right\}_{1}^{N}\right\|_{F_{\tau}(E)}+\left\|\left\{A u_{k}\right\}_{1}^{N}\right\|_{F \tau(E)} \leq M\left[\|A \varphi\|_{E}+\left\|\varphi^{\tau}\right\|_{F \tau(E)}\right]
$$

where $M$ is independent not only of $\varphi, \varphi_{\tau}$ but also of $\tau$.

Since the nonlocal boundary value problem (1.1) in the space $C(E)$ of continuous functions defined on $[0,1]$ and with values in $E$ is not well posed for the general positive operator $A$ and space $E$, then the well-posedness of the difference boundary value problem (1.2) in $C_{\tau}(E)$-norm does not take place uniformly with respect to $\tau>0$. This means that the coercive norm

$$
\left\|\left\{\tau^{-1}\left(u_{k}-u_{k-1}\right)\right\}_{1}^{N}\right\|_{F \tau(E)}+\left\|\left\{A u_{k}\right\}_{1}^{N}\right\|_{F \tau(E)}
$$

tends to $\infty$ as $\tau \rightarrow 0+$. The investigation of the difference problem (1.2) permits us to establish the order of growth of this norm to $\infty$.

Theorem 2.2. Let $\tau$ be a sufficiently small number. Then, for solution of the difference problem (1.2), obey the almost coercive inequality

$$
\left\|u^{\tau}\right\|_{K_{\tau}(E)} \leq M\left[\min \left\{\ln \frac{1}{\tau}, 1+\left|\ln \|A\|_{E \rightarrow E}\right|\right\}\right]\left\|\varphi^{\tau}\right\|_{C_{\tau}(E)}+\|A \varphi\|_{E}
$$

Proof. The almost coercive stability

$$
\left\|u^{\tau}\right\|_{K_{\tau}(E)} \leq M \min \left\{\ln \frac{1}{\tau}, 1+\left|\ln \|A\|_{E \rightarrow E}\right|\left\|\varphi^{\tau}\right\|_{C_{\tau}(E)}+\left\|A u_{0}\right\|_{E}\right\}
$$

for the solution of the initial value problem for difference (2.3) was obtained in [14]. Therefore, the proof of Theorem 2.2 is based on the estimate

$$
\left\|A u_{0}\right\|_{E} \leq M\left[\|A \varphi\|_{E}+\min \left\{\ln \frac{1}{\tau}, 1+\left|\ln \|A\|_{E \rightarrow E}\right|\right\}\left\|\varphi^{\tau}\right\|_{C_{\tau}(E)}\right]
$$

for the solution of the boundary value problem for difference (1.2), when $\tau$ is a sufficiently small number. From the strong positivity $A$, it follows that

$$
u_{0}=T\left\{\sum_{j=1}^{[\lambda / \tau]} R^{[\lambda / \tau]-j+1} \varphi_{j} \tau+\varphi\right\}
$$


276 On well-posedness of the nonlocal boundary value problem

for the solution of the boundary value problem for difference (2.3). Here,

$$
T=\left(I-R^{[\lambda / \tau]}\right)^{-1}, \quad R=(I+\tau A)^{-1} .
$$

Let $\tau$ be a sufficiently small number. Then, using the estimates

$$
\left\|(k \tau A)^{\alpha} R^{k}\right\|_{E \rightarrow E} \leq M, \quad 0 \leq \alpha \leq 1, k \geq 1,
$$

and the identity

$$
\begin{aligned}
T= & \left(I-\exp \{-\lambda A\}^{-1}\right)\left(I-R^{[\lambda / \tau]}\right)^{-1} \\
& \times \int_{0}^{1}(I+\tau s A)^{-[\lambda / \tau]-1}\left(\left(\lambda-\left[\frac{\lambda}{\tau}\right] \tau\right) A+\lambda s A^{2}\right) \exp \{-\lambda(1-s) A\} d s,
\end{aligned}
$$

we obtain the estimate

$$
\|T\|_{E \rightarrow E} \leq M
$$

Now, using formula (2.11) and the estimate (2.15), we obtain the estimate

$$
\begin{aligned}
\left\|A u_{0}\right\|_{E} & \leq\|T\|_{E \rightarrow E}\left\{\sum_{j=1}^{[\lambda / \tau]}\left\|A R^{[\lambda / \tau]-j+1}\right\|\left\|_{E \rightarrow E}\right\| \varphi_{j}\left\|_{E}+\right\| A \varphi \|_{E}\right\} \\
& \leq M_{1}\left[\sum_{j=1}^{[\lambda / \tau]}\left\|A R^{[\lambda / \tau]-j+1}\right\|_{E \rightarrow E}\left\|\varphi^{\tau}\right\|_{C_{\tau}(E)}+\|A \varphi\|_{E}\right] .
\end{aligned}
$$

Finally, using the estimate (see [14])

$$
\sum_{j=1}^{[\lambda / \tau]}\left\|A R^{[\lambda / \tau]-j+1}\right\|_{E \rightarrow E} \leq M \min \left\{\ln \frac{1}{\tau}, 1+\left|\ln \|A\|_{E \rightarrow E}\right|\right\},
$$

we obtain the estimate (2.10). Theorem 2.2 is proved.

We denote $E_{\alpha}=E_{\alpha}(A, E)$ as the fractional spaces consisting of all $v \in E$ for which the following norm is finite:

$$
\|v\|_{E_{\alpha}}=\sup _{\lambda>0} \lambda^{\alpha}\left\|A(\lambda+A)^{-1} v\right\|_{E}
$$


THeOREM 2.3. Let $\tau$ be a sufficiently small number. Then the coercivity inequality holds:

$$
\left\|\left\{\frac{u_{k}-u_{k-1}}{\tau}\right\}_{1}^{N}\right\|_{C_{\tau}\left(E_{\alpha}\right)}+\left\|A u^{\tau}\right\|_{C_{\tau}\left(E_{\alpha}\right)} \leq \frac{M}{\alpha(1-\alpha)}\left\|\varphi^{\tau}\right\|_{C_{\tau}\left(E_{\alpha}\right)}+\|A \varphi\|_{E_{\alpha}},
$$

where $M$ does not depend on $\varphi, \varphi_{k}, 1 \leq k \leq N, \alpha$, and $\tau$.

Proof. The proof of this theorem is based on the abstract theorem on the well-posedness $C_{\tau}\left(E_{\alpha}\right)$ of the initial value difference problem (2.3) of [9] and on the estimate

$$
\left\|A u_{0}\right\|_{E_{\alpha}} \leq \frac{M}{\alpha(1-\alpha)}\left\|\varphi^{\tau}\right\|_{C_{\tau}\left(E_{\alpha}\right)}+\|A \varphi\|_{E_{\alpha}}
$$

for the solution of problem (1.2), when $\tau$ is a sufficiently small number. By formula (2.11),

$$
A u_{0}=T \sum_{j=1}^{[\lambda / \tau]} A R^{[\lambda / \tau]-j+1} \varphi_{j} \tau+T A \varphi=A v_{0}+T A \varphi
$$

We estimate the norm of each term. Using the estimate (2.15), we obtain

$$
\|T A \varphi\|_{E_{\alpha}} \leq\|T\|_{E \rightarrow E}\|A \varphi\|_{E_{\alpha}} \leq M\|A \varphi\|_{E_{\alpha}} .
$$

To estimate $A v_{0}$ in the norm of $E_{\alpha}$, we use the following Cauchy-Riesz representation formula for the operator $A(\lambda+A)^{-1} R^{[\lambda / \tau]-j+1}$ (see, e.g., [11]):

$$
\lambda^{\alpha} A(\lambda+A)^{-1} A R^{[\lambda / \tau]-j+1} \varphi_{j}=\frac{1}{2 \pi i} \int_{S_{1} \cup S_{2}} \frac{z \lambda^{\alpha}}{(1+z)^{[\lambda / \tau]-j+1}} \frac{\tau}{\lambda \tau+z} A(z-\tau A)^{-1} \varphi_{j}
$$

where $S_{1}=\left\{\rho e^{i \psi}, 0 \leq \rho<\infty\right\}$ and $S_{2}=\left\{\rho e^{i \psi}, 0 \leq \rho<\infty\right\}, 0 \leq \psi<\pi / 2$. We obtain

$$
A v_{0}=\frac{T}{2 \pi i} \int_{S_{1} \cup S_{2}} \sum_{j=1}^{[\lambda / \tau]} \frac{z \lambda^{\alpha}}{(1+z)^{[\lambda / \tau]-j+1}} \frac{\tau}{\lambda \tau+z} A(z-\tau A)^{-1} \varphi_{j} \tau
$$

Since $z=\rho e^{i \psi}$, with $|\psi|<\pi / 2$, from the strong positivity of $A$, it follows that

$$
\begin{gathered}
\left(\frac{z}{\tau}\right)^{\alpha}\left\|A\left(\frac{z}{\tau}-A\right)^{-1} \varphi_{j}\right\|_{E} \leq M\left(\frac{\rho}{\tau}\right)^{\alpha}\left\|A\left(\frac{\rho}{\tau}+A\right)^{-1} \varphi_{j}\right\|_{E}, \\
\frac{1}{|\lambda \tau+z|} \leq \frac{M}{\lambda \tau+\rho} .
\end{gathered}
$$


278 On well-posedness of the nonlocal boundary value problem

Hence,

$$
\begin{aligned}
& \left\|\lambda^{\alpha} A(\lambda+A)^{-1} A v_{0}\right\|_{E} \\
& \quad \leq M\|T\|_{E \rightarrow E} \int_{0}^{\infty} \sum_{j=1}^{[\lambda / \tau]} \frac{\rho^{1-\alpha}}{\left(1+2 \rho \cos \psi+\rho^{2}\right)^{([\lambda / \tau]-j+1) / 2}} \frac{(\tau \lambda)^{\alpha}}{\lambda \tau+\rho} d \rho\left\|\varphi^{\tau}\right\|_{C_{\tau}\left(E_{\alpha}\right)} .
\end{aligned}
$$

Summing the geometric progression and using the estimate (2.15), we get

$$
\begin{aligned}
& \left\|\lambda^{\alpha} A(\lambda+A)^{-1} A v_{0}\right\|_{E} \\
& \quad \leq M_{1} \int_{0}^{\infty} \frac{\rho^{1-\alpha}}{\left(1+2 \rho \cos \psi+\rho^{2}\right)^{1 / 2}}\left[1-\frac{1}{\left(1+2 \rho \cos \psi+\rho^{2}\right)^{1 / 2}}\right] \frac{(\tau \lambda)^{\alpha}}{\lambda \tau+\rho} d \rho\left\|\varphi^{\tau}\right\|_{C_{\tau}\left(E_{\alpha}\right)} \\
& \quad \leq \frac{M_{1}}{\cos \psi} \int_{0}^{\infty} \frac{(\tau \lambda)^{\alpha}}{\rho^{\alpha}(\lambda \tau+\rho)} d \rho\left\|\varphi^{\tau}\right\|_{C_{\tau}\left(E_{\alpha}\right)} \leq \frac{M(\psi)}{\alpha(1-\alpha)}\left\|\varphi^{\tau}\right\|_{C_{\tau}\left(E_{\alpha}\right)} .
\end{aligned}
$$

Finally, using the triangle inequality, the last estimate, and (2.22), we obtain the estimate (2.20). Theorem 2.3 is proved.

THeOREM 2.4. Let $\tau$ be a sufficiently small number. Then the boundary value problem (1.2) is coercive stable in $C_{\tau}^{\alpha}(E)$.

Proof. The proof of this theorem is based on the abstract theorem on the well-posedness in $C_{\tau}^{\alpha}(E)$ of the initial value difference problem (2.3) of [14] and on the estimate

$$
\left\|A u_{0}\right\|_{E} \leq \frac{M}{\alpha(1-\alpha)}\left\|\varphi^{\tau}\right\|_{C_{\tau}^{\alpha}(E)}+M\|A \varphi\|_{E}
$$

for the solution of problem (1.2), when $\tau$ is a sufficiently small number. By formula (2.21), we have

$$
A u_{0}=A v_{0}+T A \varphi
$$

where

$$
A v_{0}=T\left\{\sum_{j=1}^{[\lambda / \tau]} A R^{[\lambda / \tau]-j+1}\left(\varphi_{j}-\varphi_{[\lambda / \tau]}\right) \tau\right\}+\varphi_{[\lambda / \tau]}
$$

We estimate the norm of each term. Using the estimate (2.15), we obtain

$$
\|T A \varphi\|_{E} \leq\|T\|_{E \rightarrow E}\|A \varphi\|_{E} \leq M\|A \varphi\|_{E}
$$


Using the estimates (2.13) and (2.15), we obtain

$$
\begin{aligned}
\left\|A v_{0}\right\|_{E} & \leq\|T\|_{E \rightarrow E}\left\{\sum_{j=1}^{[\lambda / \tau]}\left\|A R^{[\lambda / \tau]-j+1}\right\|_{E \rightarrow E}\left\|\varphi_{j}-\varphi_{[\kappa / \tau]}\right\|_{E} \tau\right\}+\left\|\varphi_{[\lambda / \tau]}\right\|_{E} \\
& \leq M\left\{\sum_{j=1}^{[\lambda / \tau]} \frac{\tau}{(([\lambda / \tau]-j+1) \tau)^{1-\alpha}(j \tau)^{\alpha}}+1\right\}\left\|\varphi^{\tau}\right\|_{C_{\tau}^{\alpha}(E)} \\
& \leq \frac{M_{1}}{\alpha(1-\alpha)}\left\|\varphi^{\tau}\right\|_{C_{\tau}^{\alpha}(E) .}
\end{aligned}
$$

Finally, using the triangle inequality, the last estimate, and (2.30), we obtain the estimate (2.28). Theorem 2.4 is proved.

Remark 2.5. By passing to the limit for $\tau \rightarrow 0$, we obtain the well-posedness of the boundary value problem (1.1) in the spaces of smooth functions.

Remark 2.6. Applying the method of the present paper and of [3], we can obtain similar results for solutions of the difference scheme of the second-order accuracy

$$
\begin{gathered}
\frac{u_{k}-u_{k-1}}{\tau}+A\left(I+\frac{\tau A}{2}\right) u_{k}=\left(I+\frac{\tau A}{2}\right) \varphi_{k}, \quad \varphi_{k}=f\left(t_{k}-\frac{\tau}{2}\right), 1 \leq k \leq N \\
u_{0}=\left(I-\left(\lambda-\left[\frac{\lambda}{\tau}\right] \tau\right) A\right) u_{[\lambda / \tau]}+\varphi+\left(\lambda-\left[\frac{\lambda}{\tau}\right] \tau\right) \varphi[\lambda / \tau]
\end{gathered}
$$

for approximate solution of problem (1.1).

Remark 2.7. Using this approach, we can obtain the same results for solutions of firstorder accuracy implicit Rothe difference scheme

$$
\begin{gathered}
\frac{u_{k}-u_{k-1}}{\tau}+A u_{k}=\varphi_{k}, \quad \varphi_{k}=f\left(t_{k}\right), 1 \leq k \leq N, N \tau=1, \\
u_{0}=\sum_{i=1}^{p} c_{i} u_{\left[\lambda_{i} / \tau\right]}+\varphi
\end{gathered}
$$

for approximate solutions of the general boundary value problem

$$
v^{\prime}(t)+A v(t)=f(t), \quad 0 \leq t \leq 1, \quad v(0)=\sum_{i=1}^{p} c_{i} v\left(\lambda_{i}\right)+\varphi
$$

where $0<\lambda_{1}<\lambda_{2}<\cdots<\lambda_{p} \leq 1$, if the operator

$$
I-\sum_{i=1}^{p} c_{i} e^{-\lambda_{i} A}
$$

has a bounded inverse in $E$.

Finally, we consider the applications of these results to the parabolic equations. 


\section{Applications}

First, we consider the nonlocal boundary value problem for heat equations

$$
\begin{gathered}
\frac{\partial u}{\partial t}-a(x) \frac{\partial^{2} u}{\partial x^{2}}+\delta u=f(t, x), \quad 0<t<1,0<x<1, \\
u(0, x)=u(\lambda, x)+\varphi(x), \quad 0 \leq x \leq 1,0<\lambda \leq 1, \\
u(t, 0)=u(t, 1), \quad u_{x}(t, 0)=u_{x}(t, 1), \quad 0 \leq t \leq 1,
\end{gathered}
$$

where $a(x), f(t, x)$ are given sufficiently smooth functions, $\delta=$ const $>0$, and $a(x)>0$.

We associate with the nonlocal boundary value problem (3.1) the corresponding difference problem

$$
\begin{gathered}
\frac{1}{\tau}\left(u_{k}^{n}-u_{k-1}^{n}\right)-a^{n} \frac{1}{h^{2}}\left(u_{k}^{n+1}-2 u_{k}^{n}+u_{k}^{n-1}\right)+\delta u_{k}^{n}=\varphi_{k}^{n}, \\
\varphi_{k}^{n}=f\left(t_{k}, x_{n}\right), \quad a^{n}=a\left(x_{n}\right), \quad t_{k}=k \tau, \\
x_{n}=n h, \quad 1 \leq k \leq N, 1 \leq n \leq M-1, \quad N \tau=1, \quad M h=1, \\
u_{0}^{n}=u_{[\lambda / \tau]}^{n}+\varphi^{n}, \quad 0 \leq n \leq M, \\
u_{k}^{n}=u_{k}^{M}, \quad u_{k}^{1}-u_{k}^{0}=u_{k}^{M}-u_{k}^{M-1}, \quad 0 \leq k \leq N .
\end{gathered}
$$

We introduce the Banach spaces $C_{h}, C_{h}^{\beta}$ of grid functions $\varphi^{h}(x)=\left\{\varphi^{n}\right\}_{1}^{M-1}$ with norms

$$
\begin{aligned}
\left\|\varphi^{h}\right\|_{C_{h}} & =\max _{1 \leq n \leq M-1}\left|\varphi^{n}\right| \\
\left\|\varphi^{h}\right\| C_{h}^{\beta} & =\left\|\varphi^{h}\right\|_{C_{h}}+\max _{1 \leq n<n+r<M} \frac{\left|\varphi^{n+r}-\varphi^{n}\right|}{(r \tau)^{\alpha}} .
\end{aligned}
$$

It is known (see $[7,8])$ that the difference operator

$$
\begin{aligned}
& A_{h}^{x} u_{h}^{x}=\left\{-a^{n} D_{h}^{2} u^{n}+\delta u^{n}\right\}_{1}^{M-1}, \\
& D_{h}^{2} u^{n}=\frac{u^{n+1}-2 u^{n}+u^{n-1}}{h^{2}}, \quad 1 \leq n \leq M-1,
\end{aligned}
$$

acting on the space of grid functions $\varphi^{h}(x)=\left\{\varphi^{n}\right\}_{0}^{M}$ satisfying the conditions $\varphi^{0}=\varphi^{M}$, $\varphi^{1}-\varphi^{0}=\varphi^{M}-\varphi^{M-1}$ is a positive operator. Therefore, we can replace the difference problem (3.2) by the abstract boundary value problem (1.2). Using the results of $[7,8]$ and of Theorems 2.1, 2.2, 2.3, and 2.4, we obtain the following result. 
TheOREM 3.1. Let $\tau$ be a sufficiently small number. Then, for the solution of the difference problem (3.2), the following inequalities are valid:

$$
\begin{aligned}
& \left\|\left(u^{h}\right)^{\tau}\right\|_{C_{\tau}^{\alpha}\left(C_{h}^{\beta}\right)} \leq M_{1}\left[\left\|\left(\varphi^{h}\right)^{\tau}\right\|_{C_{\tau}^{\alpha}\left(C_{h}^{\beta}\right)}+\left\|\varphi^{h}\right\|_{C_{h}^{\beta}}\right], \quad 0 \leq \alpha<1, \beta \geq 0, \\
& \left\|\left\{\left\{\frac{1}{\tau}\left(u_{k}^{n}-u_{k-1}^{n}\right)\right\}_{1}^{M-1}\right\}_{1}^{N}\right\|_{C_{\tau}\left(C_{h}\right)}+\left\|\left\{\left\{D_{h}^{2} u_{k}^{n}\right\}_{1}^{M-1}\right\}_{1}^{N}\right\|_{C_{\tau}\left(C_{h}\right)} \\
& \quad \leq M_{1}\left[\ln \frac{1}{\tau+h}\left\|\left(\varphi^{h}\right)^{\tau}\right\|_{C_{\tau}\left(C_{h}\right)}+\left\|\left\{D_{h}^{2} \varphi^{n}\right\}_{1}^{M-1}\right\|_{C_{h}}\right], \\
& \left\|\left\{\left\{\frac{1}{\tau}\left(u_{k}^{n}-u_{k-1}^{n}\right)\right\}_{1}^{M-1}\right\}_{1}^{N}\right\|_{C_{\tau}\left(C_{h}^{\beta}\right)}+\left\|\left\{\left\{D_{h}^{2} u_{k}^{n}\right\}_{1}^{M-1}\right\}_{1}^{N}\right\|_{C_{\tau}\left(C_{h}^{\beta}\right)} \\
& \quad \leq \frac{M_{1}}{\beta(1-\beta)}\left[\left\|\left(\varphi^{h}\right)^{\tau}\right\|_{C_{\tau}\left(C_{h}^{\beta}\right)}+\left\|\left\{D_{h}^{2} \varphi^{n}\right\}_{1}^{M-1}\right\|_{C_{h}^{\beta}}\right], \quad 0<\beta<1, \\
& \left\|\left\{\left\{\frac{1}{\tau}\left(u_{k}^{n}-u_{k-1}^{n}\right)\right\}_{1}^{M-1}\right\}_{1}^{N}\right\|_{C_{\tau}^{\alpha}\left(C_{h}^{\beta}\right)}+\left\|\left\{\left\{D_{h}^{2} u_{k}^{n}\right\}_{1}^{M-1}\right\}_{1}^{N}\right\|_{C_{\tau}^{\alpha}\left(C_{h}^{\beta}\right)} \\
& \quad \leq \frac{M_{1}}{\alpha(1-\alpha)}\left[\left\|\left(\varphi^{h}\right)^{\tau}\right\|_{C_{\tau}^{\alpha}\left(C_{h}^{\beta}\right)}+\left\|\left\{D_{h}^{2} \varphi^{n}\right\}_{1}^{M-1}\right\|_{C_{h}^{\beta}}\right], \quad 0<\alpha<1, \beta \geq 0,
\end{aligned}
$$

where $M_{1}$ is independent of $\left(\varphi^{h}\right)^{\tau}, \varphi^{h}, \alpha$, and $\beta$.

Second, let $\Omega$ be the unit open cube in the $n$-dimensional Euclidean space $\mathbb{R}^{n}\left(0<x_{k}<\right.$ $1,1 \leq k \leq n)$ with boundary $S, \bar{\Omega}=\Omega \cup S$. In $[0,1] \times \Omega$ we consider the mixed boundary value problem for the multidimensional parabolic equation

$$
\begin{gathered}
\frac{\partial v(t, x)}{\partial t}-\sum_{r=1}^{n} \alpha_{r}(x) \frac{\partial^{2} v(t, x)}{\partial x_{r}^{2}}+\delta v(t, x)=f(t, x), \\
x=\left(x_{1}, \ldots, x_{n}\right) \in \Omega, \quad 0<t<1, \\
v(0, x)=v(\lambda, x)+\varphi(x), \quad x \in \bar{\Omega}, 0<\lambda \leq 1, \\
v(t, x)=0, \quad x \in S, 0 \leq r \leq n,
\end{gathered}
$$

where $\alpha_{r}(x)(x \in \Omega)$ and $f(t, x)(t \in(0,1), x \in \Omega)$ are given smooth functions, $\alpha_{r}(x)>0$, and $\delta>0$ is a sufficiently large number.

We introduce the Banach spaces $C_{01}^{\beta}(\bar{\Omega})\left(\beta=\left(\beta_{1}, \ldots, \beta_{n}\right), x \in \Omega\right)$ obtained by completion of the space of all continuous functions on $\bar{\Omega}$ in the norm

$$
\begin{aligned}
\|f\|_{C_{01}^{\beta}(\bar{\Omega})}= & \|f\|_{C(\bar{\Omega})}+\sup _{x \in \bar{\Omega}}\left|f\left(x_{1}, x_{2}, \ldots, x_{n}\right)-f\left(x_{1}+h_{1}, \ldots, x_{n}+h_{n}\right)\right| \\
& \times \prod_{k=1}^{n} h_{k}^{-\beta_{k}} x_{k}^{\beta_{k}}\left(1-x_{k}-h_{k}\right)^{\beta_{k}},
\end{aligned}
$$


282 On well-posedness of the nonlocal boundary value problem

where $C(\bar{\Omega})$ is the space of the all continuous functions defined on $\bar{\Omega}$, equipped with the norm

$$
\|f\|_{C(\bar{\Omega})}=\max _{x \in \bar{\Omega}}|f(x)| .
$$

It is known (see $[14,15])$ that the differential expression

$$
A^{x} u=-\sum_{r=1}^{n} \alpha_{r}(x) \frac{\partial^{2} u(x)}{\partial x_{r}^{2}}+\delta u(x)
$$

defines a positive operator $A^{x}$ acting on $C_{01}^{2+\beta}(\bar{\Omega})$ and satisfying the condition $u=0$ on $S$.

We define the grid sets

$$
\begin{gathered}
\bar{\Omega}_{h}=\left\{x=x_{j}=\left(h_{1} j_{1}, \ldots, h_{n} j_{n}\right), j=\left(j_{1}, \ldots, j_{n}\right), 0 \leq j_{r} \leq N_{r}, h_{r} N_{r}=1, r=1, \ldots, n\right\}, \\
\Omega_{h}=\bar{\Omega} h \cap \Omega, \quad S_{h}=\bar{\Omega} h \cap S .
\end{gathered}
$$

We introduce the Banach spaces $C\left(\bar{\Omega}_{h}\right), C_{01}^{\beta}\left(\bar{\Omega}_{h}\right), L_{p}\left(\bar{\Omega}_{h}\right)$ of grid functions $\varphi^{h}(x)=$ $\left\{\varphi\left(h_{1} j_{1}, \ldots, h_{n} j_{n}\right)\right\}$ defined on $\bar{\Omega}_{h}$, equipped with the norms

$$
\begin{aligned}
\left\|\varphi^{h}\right\|_{C\left(\bar{\Omega}_{h}\right)}= & \max _{x \in \bar{\Omega}_{h}}\left|\varphi^{h}(x)\right|, \\
\left\|\varphi^{h}\right\|_{C_{01}^{\beta}\left(\bar{\Omega}_{h}\right)}= & \left\|\varphi^{h}\right\|_{C\left(\bar{\Omega}_{h}\right)}+\sup _{\substack{x \in \bar{\Omega}_{h} \\
0<x_{j_{r}}+\Delta y_{j_{r}} \\
\Delta y_{j_{r}}=m_{j_{r}} h_{r} \\
1 \leq r \leq n, m_{j_{r}}=1,2, \ldots}}\left|\varphi\left(x_{j_{1}}, \ldots, x_{j_{n}}\right)-\varphi\left(x_{j_{1}}+\Delta y_{j_{1}}, \ldots, x_{j_{n}}+\Delta y_{j_{n}}\right)\right| \\
& \times \prod_{r=1}^{n}\left(\Delta y_{j_{r}}\right)^{-\beta_{r}} x_{j_{r}}^{\beta_{r}}\left(1-x_{j_{r}}-\Delta y_{j_{r}}\right)^{\beta_{r}} \quad\left(0<\beta_{r}<1,1 \leq r \leq n\right) .
\end{aligned}
$$

We define the difference operator $A_{h}^{x}$,

$$
A_{h}^{x} \varphi^{h}(x)=\left\{-\sum_{r=1}^{n} a_{r}\left(x_{j_{1}}, \ldots, x_{j_{n}}\right) D_{h_{r}}^{2} \varphi\left(x_{j_{1}}, \ldots, x_{j_{n}}\right)+\delta \varphi\left(x_{j_{1}}, \ldots, x_{j_{n}}\right)\right\}_{1}^{M-1}
$$

where

$$
\begin{aligned}
& D_{h_{r}}^{2} \varphi\left(x_{j_{1}}, \ldots, x_{j_{n}}\right)=\frac{1}{h_{r}^{2}}\left(\varphi\left(x_{j_{1}}, \ldots, x_{j_{r-1}}, x_{j_{r+1}}, x_{j_{r+1}}, \ldots, x_{j_{n}}\right)\right. \\
&-2 \varphi\left(x_{j_{1}}, \ldots, x_{j_{r-1}}, x_{j_{r}}, x_{j_{r+1}}, \ldots, x_{j_{n}}\right) \\
&\left.+\varphi\left(x_{j_{1}}, \ldots, x_{j_{r-1}}, x_{j_{r-1}}, x_{j_{r+1}}, \ldots, x_{j_{n}}\right)\right), \\
& x=\left(x_{j_{1}}, \ldots, x_{j_{n}}\right) \in \bar{\Omega}_{h}, \quad 1 \leq r \leq n,
\end{aligned}
$$

which acts on grid functions $\varphi^{h}(x)=0, x \in S_{h}$. 
The strong positivity of this elliptic difference operator $A_{h}^{x}$ in the $C_{01}^{\beta}\left(\bar{\Omega}_{h}\right)_{-}, C\left(\bar{\Omega}_{h}\right)^{-}$, $L_{p}\left(\bar{\Omega}_{h}\right)$-norms was established in $[1,2,14]$. Using $(1.2)$, we have the nonlocal difference problem

$$
\begin{gathered}
\frac{1}{\tau}\left[u_{k}^{h}(x)-u_{k-1}^{h}(x)\right]+A_{h}^{x} u_{k}^{h}(x)=\varphi_{k}^{h}(x), \\
\varphi_{k}^{h}(x)=f\left(t_{k}, x\right), \quad x \in \bar{\Omega}_{h}, 1 \leq k \leq N, \\
u_{0}^{h}(x)=u_{[\lambda / \tau]}^{h}(x)+\varphi^{h}(x), \quad x \in \bar{\Omega}_{h}, 0<\lambda \leq 1 .
\end{gathered}
$$

The abstract result of this paper and $[1,2,11,14,15]$ permits us to obtain the following result.

TheOrem 3.2. Let $\tau$ be a sufficiently small number. Then, for the solution of the difference problem (3.14), the following stability inequalities are valid:

$$
\begin{aligned}
& \left\|\left\{u_{h}^{k}(x)\right\}_{1}^{N}\right\|_{C_{T}^{\alpha}\left(C_{01}^{\beta}\left(\overline{\Omega_{h}}\right)\right)} \leq M_{1}\left[\left\|\left\{\varphi_{k}^{h}(x)\right\}_{1}^{N}\right\|_{C_{\tau}^{\alpha}\left(C_{01}^{\beta}\left(\overline{\Omega_{h}}\right)\right)}+\left\|\varphi^{h}(x)\right\|_{C_{01}^{\beta}\left(\overline{\Omega_{h}}\right)}\right], \\
& 0 \leq \alpha<1, \quad \beta=\left(\beta_{1}, \ldots, \beta_{n}\right), \quad \beta_{r} \geq 0, \quad 1 \leq r \leq n, \\
& \left\|\left\{u_{h}^{k}(x)\right\}_{1}^{N}\right\|_{C_{\tau}^{\alpha}\left(L_{p}\left(\overline{\Omega_{h}}\right)\right)} \leq M_{1}\left[\left\|\left\{\varphi_{k}^{h}(x)\right\}_{1}^{N}\right\|_{C_{\tau}^{\alpha}\left(L_{p}\left(\overline{\Omega_{h}}\right)\right)}+\left\|\varphi^{h}(x)\right\|_{L_{p}\left(\overline{\Omega_{h}}\right)}\right] \text {, } \\
& 0 \leq \alpha<1, \quad 1 \leq p<\infty \text {, } \\
& \left\|\left\{\frac{1}{\tau}\left(u_{h}^{k}(x)-u_{h-1}^{k}(x)\right)\right\}_{1}^{N}\right\|_{C_{\tau}\left(C\left(\bar{\Omega}_{h}\right)\right)}+\sum_{r=1}^{n}\left\|\left\{D_{h_{r}}^{2} u_{k}^{h}(x)\right\}_{1}^{N}\right\|_{C_{\tau}\left(C\left(\bar{\Omega}_{h}\right)\right)} \\
& \leq M_{1}\left[\ln \frac{1}{\tau+|h|}\left\|\left\{\varphi_{h}^{k}(x)\right\}_{1}^{N}\right\|_{C_{\tau}\left(C\left(\bar{\Omega}_{h}\right)\right)}+\sum_{r=1}^{n}\left\|\left\{D_{h_{r}}^{2} \varphi^{h}(x)\right\}\right\|_{C\left(\bar{\Omega}_{h}\right)}\right], \\
& |h|=h_{1}+\cdots+h_{n}, \\
& \left\|\left\{\frac{1}{\tau}\left(u_{k}^{h}(x)-u_{k-1}^{h}(x)\right)\right\}_{1}^{N}\right\|_{C_{\tau}^{\alpha}\left(C_{01}^{\beta}\left(\overline{\Omega_{h}}\right)\right)}+\sum_{r=1}^{n}\left\|\left\{D_{h_{r}}^{2} u_{k}^{h}(x)\right\}_{1}^{N}\right\|_{C_{\tau}^{\alpha}\left(C_{01}^{\beta}\left(\overline{\Omega_{h}}\right)\right)} \\
& \leq M(\alpha, \beta)\left[\left\|\left\{\varphi_{k}^{h}(x)\right\}_{1}^{N}\right\|_{C_{\tau}^{\alpha}\left(C_{01}^{\beta}\left(\overline{\Omega_{h}}\right)\right)}+\sum_{r=1}^{n}\left\|\left\{D_{h_{r}}^{2} \varphi^{h}(x)\right\}\right\|_{C_{01}^{\beta}\left(\overline{\Omega_{h}}\right)}\right], \\
& 0<\alpha<1, \quad \beta=\left(\beta_{1}, \ldots, \beta_{n}\right), \quad \beta_{r}>0, \quad 1 \leq r \leq n,
\end{aligned}
$$

where $M_{1}$ is independent of $\left\{\varphi_{k}^{h}(x)\right\}_{1}^{N}, \varphi^{h}(x), \alpha, \beta, p$, and $M(\alpha, \beta)$ is independent of $\left\{\varphi_{k}^{h}(x)\right\}_{1}^{N}, \varphi^{h}(x)$.

Third, we consider the boundary value problem on the range $\left\{0 \leq t \leq 1, x \in \mathbb{R}^{n}\right\}$ for $2 m$-order multidimensional differential equations of parabolic type:

$$
\begin{gathered}
\frac{\partial v(t, x)}{\partial t}+\sum_{\tau=2 m} a_{r}(x) \frac{\partial^{|\tau|} v(t, x)}{\partial x_{1}^{\tau_{1}} \cdots \partial x_{n}^{\tau_{n}}}+\delta v(t, x)=f(t, x), \quad 0 \leq t \leq 1, \\
v(0, x)=v(\lambda, x)+\varphi(x), \quad 0<\lambda \leq 1, x \in \mathbb{R}^{n},|\tau|=\tau_{1}+\cdots+\tau_{n},
\end{gathered}
$$


where $a_{r}(x), f(t, x)$ are given sufficiently smooth functions and $\delta>0$ is a sufficiently large number. We will assume that the symbol

$$
B(\xi)=\sum_{|\tau|=2 m} a_{r}(x)(i \xi)^{r_{1}} \cdots(i \xi)^{r_{n}}
$$

of the differential operator of the form

$$
B^{x}=\sum_{|r=2 m|} a_{r}(x) \frac{\partial^{|r|}}{\partial x_{1}^{r_{1}} \cdots \partial x_{n}^{r_{n}}}
$$

acting on functions defined on the space $\mathbb{R}^{n}$ satisfies the inequalities

$$
0<M_{1}|\xi|^{2 m} \leq(-1)^{m} B(\xi) \leq M_{2}|\xi|^{2 m}<\infty
$$

for $\xi \neq 0$.

Problem (3.16) has a unique smooth solution. This allows us to reduce the boundary value problem (3.16) to the boundary value problem (1.1) in Banach space $E$ with a strongly positive operator $A^{x}=B^{x}+\delta I$ defined by (3.18). We define the grid space $\mathbb{R}_{h}^{n}$ $\left(0 \leq h \leq h_{0}\right)$ as the set of all points of the space $\mathbb{R}^{n}$ whose coordinates are given by

$$
x_{k}=m_{k}^{h}, \quad m_{k}=0, \pm 1, \pm 2, \ldots, \quad 1 \leq k \leq n .
$$

We introduce the spaces $C\left(\mathbb{R}_{h}^{n}\right), L_{p}\left(\mathbb{R}_{h}^{n}\right), C_{\beta}\left(\mathbb{R}_{h}^{n}\right)$ of all grid functions $u^{h}(x)$, defined by the norms

$$
\begin{aligned}
& \left\|u^{h}\right\|_{C\left(\mathbb{R}_{h}^{n}\right)}=\sup _{x \in \mathbb{R}_{h}^{n}}\left|u^{h}(x)\right|, \\
& \left\|u^{h}\right\|_{L_{p}\left(\mathbb{R}_{h}^{n}\right)}=\left(\sum_{x \in \mathbb{R}_{h}^{n}}\left|u^{h}(x)\right|^{p} h^{n}\right)^{1 / p}, \quad 1 \leq p<\infty, \\
& \left\|u^{h}\right\|_{C^{\beta}\left(\mathbb{R}_{h}^{n}\right)}=\left\|u^{h}\right\|_{C\left(\mathbb{R}_{h}^{n}\right)}+\sup _{\substack{x, y \in \mathbb{R}_{h}^{n} \\
x \neq y}} \frac{\left|u^{h}(x)-u^{h}(y)\right|}{|x-y|^{\beta}}, \quad 0 \leq \beta<1 .
\end{aligned}
$$

By replacing the differential operator $A^{x}$ with the difference operator $A_{h}^{x}$ that acts on the Banach spaces $C\left(\mathbb{R}_{h}^{n}\right), L_{p}\left(\mathbb{R}_{h}^{n}\right), C^{\beta}\left(\mathbb{R}_{h}^{n}\right)$ and is uniformly strongly positive in $h$ for $0 \leq h \leq h_{0}$, and using (1.2), we have the nonlocal difference problem

$$
\begin{gathered}
\frac{1}{\tau}\left[u_{k}^{h}(x)-u_{k-1}^{h}(x)\right]+A_{h}^{x} u_{k}^{h}(x)=\varphi_{k}^{h}(x), \quad \varphi_{k}^{h}(x)=f\left(t_{k}, x\right), x \in \mathbb{R}_{h}^{n}, 1 \leq k \leq N, \\
u_{0}^{h}(x)=u_{[\lambda / \tau]}^{h}(x)+\varphi^{h}(x), \quad x \in \mathbb{R}_{h}^{n}, 0<\lambda \leq 1 .
\end{gathered}
$$

The abstract result of this paper and of $[11,13]$ permits us to obtain the stability in $C_{\tau}^{\alpha}\left(C^{\beta}\left(\mathbb{R}_{h}^{n}\right)\right)(0 \leq \beta \leq 1 / 2 m, 0 \leq \alpha<1), C_{\tau}\left(L_{p}\left(\mathbb{R}_{h}^{n}\right)\right)(1 \leq p<\infty)$-norms, the almost coercive stability in $C_{\tau}\left(C\left(\mathbb{R}_{h}^{n}\right)\right)$-norm with the multiplier $\ln (1 /(\tau+h))$, and the coercive stability in $C_{\tau}^{\alpha}\left(C^{\beta}\left(\mathbb{R}_{h}^{n}\right)\right)(0<\beta<1 / 2 m, 0<\alpha<1)$-norm of the nonlocal boundary difference problem (3.22). 
Table 4.1. The first line is the exact solution, the second line is the solution of Rothe's difference scheme, and the third line is the solution of the second-order accuracy difference scheme.

\begin{tabular}{ccccccc}
\hline$t_{k} \backslash x_{n}$ & 0.0 & 0.2 & 0.4 & 0.6 & 0.8 & 1.0 \\
\hline 0.0 & 0 & 0 & 0 & 0 & 0 & 0 \\
& 0 & -0.0019 & -0.0031 & -0.0031 & -0.0019 & 0 \\
& 0 & 0.0014 & 0.0023 & 0.0023 & 0.0014 & 0 \\
\hline 0.2 & 0 & 0.0940 & 0.1522 & 0.1522 & 0.0940 & 0 \\
& 0 & 0.0921 & 0.1491 & 0.1491 & 0.0921 & 0 \\
& 0 & 0.0934 & 0.1512 & 0.1512 & 0.0934 & 0 \\
\hline 0.4 & 0 & 0.1411 & 0.2283 & 0.2283 & 0.1411 & 0 \\
& 0 & 0.1392 & 0.2252 & 0.2252 & 0.1392 & 0 \\
& 0 & 0.1408 & 0.2278 & 0.2278 & 0.1408 & 0 \\
\hline 0.6 & 0 & 0.1411 & 0.2283 & 0.2283 & 0.1411 & 0 \\
& 0 & 0.1392 & 0.2252 & 0.2252 & 0.1392 & 0 \\
& 0 & 0.1414 & 0.2278 & 0.2278 & 0.1414 & 0 \\
\hline 0.8 & 0 & 0.0940 & 0.1522 & 0.1522 & 0.0940 & 0 \\
& 0 & 0.0922 & 0.1491 & 0.1491 & 0.0922 & 0 \\
& 0 & 0.0949 & 0.1536 & 0.1536 & 0.0949 & 0 \\
\hline 1.0 & 0 & 0 & 0 & 0 & 0 & 0 \\
& 0 & -0.0019 & -0.0031 & -0.0031 & -0.0019 & 0 \\
& 0 & 0.0014 & 0.0023 & 0.0023 & 0.0014 & 0 \\
\hline
\end{tabular}

\section{Numerical analysis}

We consider the nonlocal boundary value problem

$$
\begin{gathered}
\frac{\partial u(t, x)}{\partial t}-\frac{\partial^{2} u(t, x)}{\partial x^{2}}+u(t, x)=\left[1-2 t+t(1-t)\left(\pi^{2}+1\right)\right] \sin \pi x, \\
0<x<1, \quad 0<t<1, \\
u(0, x)=u(1, x), \quad 0 \leq x \leq 1, \\
u(t, 0)=u(t, 1)=0, \quad 0 \leq t \leq 1
\end{gathered}
$$

for heat equation. The exact solution is

$$
u(t, x)=t(1-t) \sin \pi x
$$

For approximate solutions of the nonlocal boundary value problem (4.1), we will use Rothe's scheme and a second-order accuracy difference scheme with $\tau=1 / 30, h=1 / 30$. We have the second-order or fourth-order difference equations with matrix coefficients. To solve these difference equations, we have applied a procedure of modified Gauss elimination method for difference equations with matrix coefficients. The exact and numerical solutions are given in Table 4.1

Thus, the second-order accuracy difference scheme was more accurate compared with Rothe's difference scheme. 


\section{References}

[1] H. A. Alibekov and P. E. Sobolevskii, The stability of difference schemes for parabolic equations, Dokl. Akad. Nauk SSSR 232 (1977), no. 4, 737-740 (Russian).

[2] Stability and convergence of high-order difference schemes of approximation for parabolic equations, Ukrain. Mat. Zh. 31 (1979), no. 6, 627-634 (Russian).

[3] A. Ashyralyev, A purely implicit difference scheme of second order of approximation for parabolic equations, Izv. Akad. Nauk Turkmen. SSR Ser. Fiz.-Tekhn. Khim. Geol. Nauk 1987 (1987), no. 4, 3-13 (Russian).

[4] Correct solvability of Padé difference schemes for parabolic equations in Hölder spaces, Ukrain. Mat. Zh. 44 (1992), no. 11, 1466-1476 (Russian).

[5] A. Ashyralyev, H. Akca, and L. Biszewski, On a semilinear evolution nonlocal Cauchy problem, Some Problems of Applied Mathematics, Fatih University, Istanbul, 2000, pp. 29-44.

[6] A. Ashyralyev, A. Hanalyev, and P. E. Sobolevskii, Coercive solvability of the nonlocal boundary value problem for parabolic differential equations, Abstr. Appl. Anal. 6 (2001), no. 1, 53-61.

[7] A. Ashyralyev and B. Kendirli, Positivity in $C_{h}$ of one dimensional difference operators with nonlocal boundary conditions, Some Problems of Applied Mathematics, Fatih University, Istanbul, 2000, pp. 45-60.

[8] Positivity in Hölder norms of one-dimensional difference operators with nonlocal boundary conditions, Applications of Mathematics in Engineering and Economics (Sozopol, 2000), Heron Press, Sofia, 2001, pp. 134-137.

[9] A. Ashyralyev and P. E. Sobolevskii, The theory of interpolation of linear operators and the stability of difference schemes, Dokl. Akad. Nauk SSSR 275 (1984), no. 6, 1289-1291 (Russian).

[10] Difference schemes of a high order of accuracy for parabolic equations with variable coefficients, Dokl. Akad. Nauk Ukrain. SSR Ser. A 1988 (1988), no. 6, 3-7 (Russian).

[11] Well-Posedness of Parabolic Difference Equations, Operator Theory: Advances and Applications, vol. 69, Birkhäuser, Basel, 1994.

[12] Well-posed solvability of the Cauchy problem for difference equations of parabolic type, Nonlinear Anal. 24 (1995), no. 2, 257-264.

[13] Y. A. Smirnitskii and P. E. Sobolevskii, Positivity of multidimensional difference operators in the C-norm, Uspekhi Mat. Nauk 36 (1981), no. 4, 202-203 (Russian).

[14] P. E. Sobolevskii, The coercive solvability of difference equations, Dokl. Akad. Nauk SSSR 201 (1971), 1063-1066 (Russian).

[15] _ The theory of semigroups and the stability of difference schemes, Operator Theory in Function Spaces (Proc. School, Novosibirsk, 1975), Izdat. "Nauka” Sibirsk. Otdel., Novosibirsk, 1977, pp. 304-337.

A. Ashyralyev: Department of Mathematics, Fatih University, 34900 Büyükçekmece, Istanbul, Turkey

E-mail address: aashyr@fatih.edu.tr

Current address: Department of Applied Mathematics, International Turkmen-Turkish University,

32 Gerogly Street, 74400 Ashgabat, Turkmenistan

I. Karatay: Department of Mathematics, Fatih University, 34900 Büyükçekmece, Istanbul, Turkey

E-mail address: ikaratay@fatih.edu.tr

Current address: Department of Mathematics, Yildiz Technical University, Davutpasa Campus, 34210 Esenler, Istanbul, Turkey

P. E. Sobolevskii: Institute of Mathematics, Hebrew University, Givat Ram 91904, Jerusalem, Israel E-mail address: pavels@math.huji.ac.il 


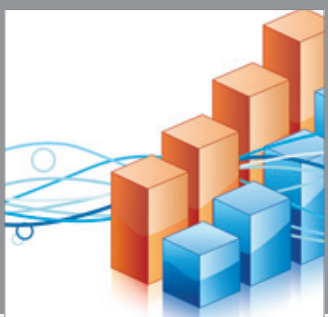

Advances in

Operations Research

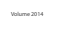

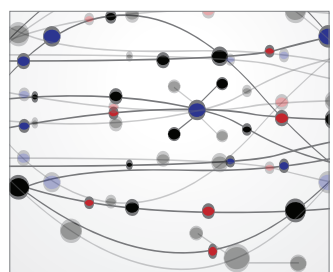

\section{The Scientific} World Journal
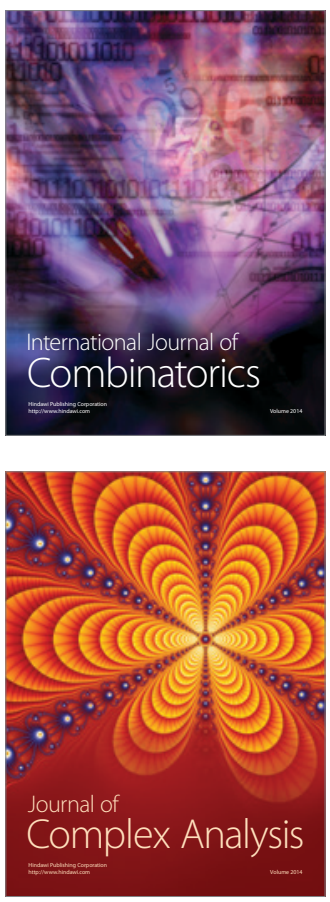

International Journal of

Mathematics and

Mathematical

Sciences
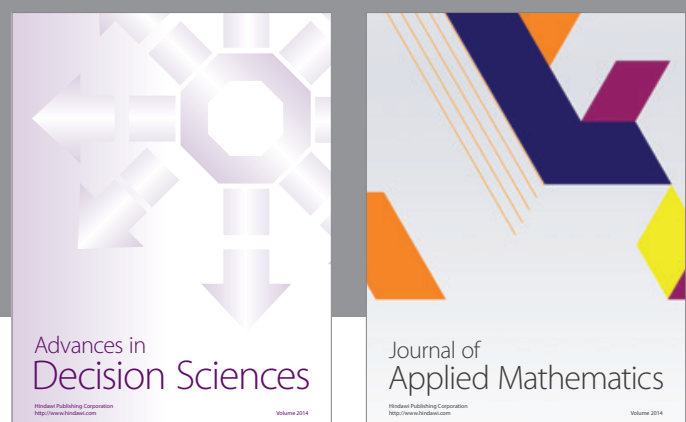

Journal of

Applied Mathematics
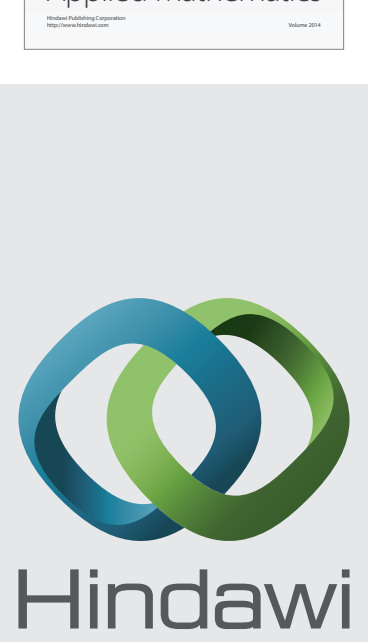

Submit your manuscripts at http://www.hindawi.com
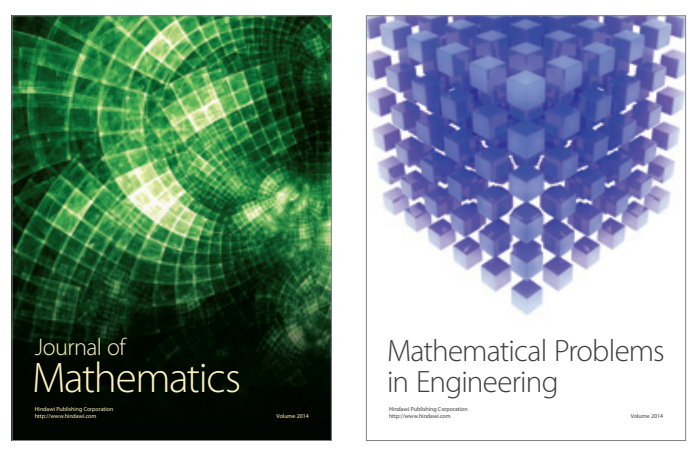

Mathematical Problems in Engineering
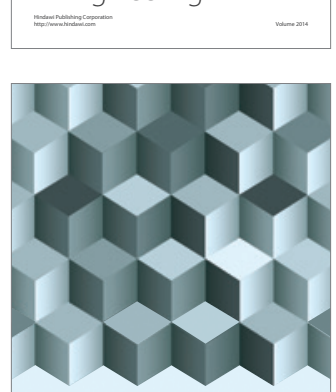

Journal of

Function Spaces
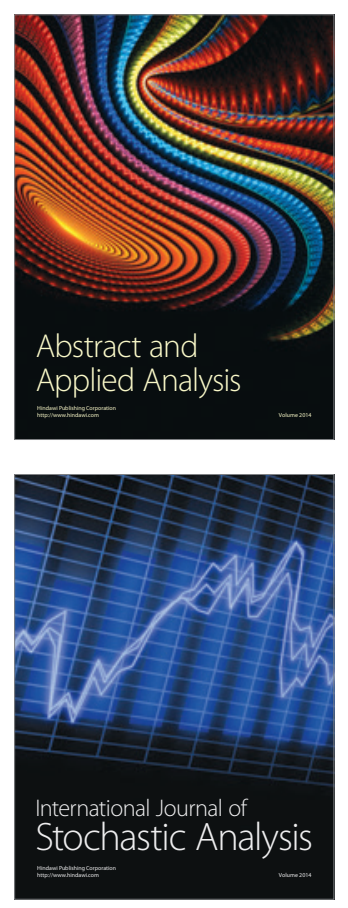

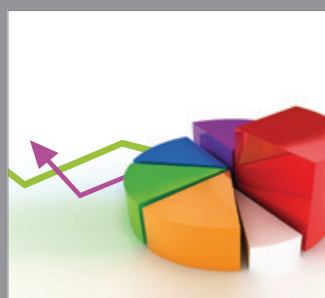

ournal of

Probability and Statistics

Promensencen
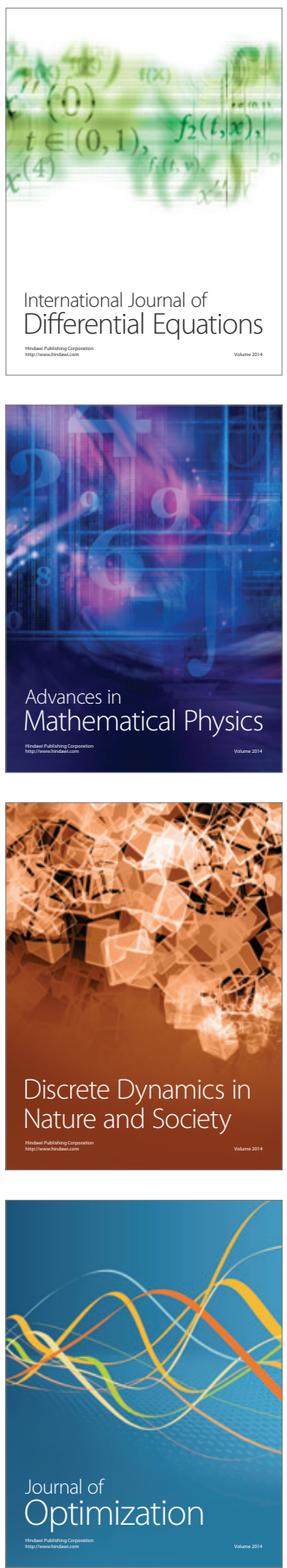